PROCEEDINGS OF THE

AMERICAN MATHEMATICAL SOCIETY

Volume 139, Number 10, October 2011, Pages 3527-3535

S 0002-9939(2011)10884-0

Article electronically published on February 18, 2011

\title{
MULTIPLE SOLUTIONS FOR NONLINEAR NEUMANN PROBLEMS DRIVEN BY A NONHOMOGENEOUS DIFFERENTIAL OPERATOR
}

\author{
D. MOTREANU AND N. S. PAPAGEORGIOU
}

(Communicated by Walter Craig)

\begin{abstract}
We consider a nonlinear Neumann problem driven by a nonhomogeneous quasilinear degenerate elliptic differential operator div $a(x, \nabla u)$, a special case of which is the $p$-Laplacian. The reaction term is a Carathéodory function $f(x, s)$ which exhibits subcritical growth in $s$. Using variational methods based on the mountain pass and second deformation theorems, together with truncation and minimization techniques, we show that the problem has three nontrivial smooth solutions, two of which have constant sign (one positive, the other negative). A crucial tool in our analysis is a result of independent interest which we prove here and which relates $W^{1, p}$ and $C^{1}$ local minimizers of a $C^{1}$-functional constructed with the general differential operator $\operatorname{div} a(x, \nabla u)$.
\end{abstract}

\section{INTRODUCTION}

Let $\Omega$ be a bounded domain in $\mathbb{R}^{N}$ with a $C^{2}$-boundary $\partial \Omega$. In this paper, we study the following nonlinear Neumann problem:

$$
\begin{cases}-\operatorname{div} a(x, \nabla u(x))=f(x, u(x)) & \text { in } \Omega, \\ \frac{\partial u}{\partial n}=0 & \text { on } \partial \Omega,\end{cases}
$$

where $\frac{\partial u}{\partial n}$ stands for the normal derivative of $u$ on $\partial \Omega$. Here $a: \bar{\Omega} \times \mathbb{R}^{N} \rightarrow \mathbb{R}^{N}$ is a continuous map such that, for every $x \in \bar{\Omega}, a(x, \cdot)$ is strictly monotone on $\mathbb{R}^{N}$, and $(x, y) \mapsto a(x, y)$ is $C^{1}$ on $\bar{\Omega} \times\left(\mathbb{R}^{N} \backslash\{0\}\right)$. The precise hypotheses on $a(x, y)$ are formulated in $\mathrm{H}(a)$ in Section 2 and incorporate as a special case the $p$-Laplace differential operator. Hypothesis $\mathrm{H}(a)$ is used in many works (see, e.g., [5, 6, 9, 14, 22]). Specifically, it was introduced to address situations beyond the $p$-Laplacian case, involving quasilinear, possibly degenerate, elliptic operators, not necessarily $(p-1)$-homogeneous. For instance, Example $2.2\left(e_{4}\right)$ in Section 2 sets forth the sum of the $p$-Laplacian and the generalized mean curvature operator.

In (1.1), the reaction $f(x, s)$ is a Carathéodory function (i.e., for all $s \in \mathbb{R}$, $x \mapsto f(x, s)$ is measurable on $\Omega$ and, for a.a. $x \in \Omega, s \mapsto f(x, s)$ is continuous) with subcritical growth. The precise hypotheses on $f(x, s)$ are given in $\mathrm{H}(f)$ in

Received by the editors March 17, 2010 and, in revised form, July 23, 2010 and August 24, 2010.

2010 Mathematics Subject Classification. Primary 35J40; Secondary 35J70.

Key words and phrases. Nonlinear Neumann problem, $p$-Laplacian, local minimizers, mountain pass theorem, second deformation theorem, nonlinear regularity theory. 
Section 4. In particular, they ensure that the energy functional of problem (1.1) is coercive.

Under our hypotheses, problem (1.1) admits the trivial solution, so the real challenge is to establish the existence of nontrivial solutions, actually of multiple solutions, and to point out qualitative properties of the solutions (as sign information). Our purpose is to prove a "three solutions theorem" for problem (1.1). Recently, such multiplicity results were proved for the coercive Dirichlet problem driven by the $p$-Laplacian in 4, 12, 13, 16, 17, 20, 23, (see also [5, 9, for slightly more general differential operators producing two constant sign solutions). No such results exist for the Neumann problem. Some recent multiplicity results for the Neumann problem deal with equations involving the $p$-Laplacian, but they impose the restrictive condition $p>N$ or are related to parameters (see [2, 7, 19, 21]).

Our main result, stated in Theorem 4.2, provides three nontrivial smooth solutions for problem (1.1), two of which are of opposite constant sign.

The approach relies on variational methods involving the mountain pass theorem and the second deformation theorem, combined with truncation and minimization techniques. A crucial tool in our analysis is a result of independent interest, namely Theorem 3.1, describing the relationship between the $W^{1, p}$ and $C^{1}$ local minimizers for $C^{1}$-functions whose pattern is the Euler functional associated to problem (1.1). Theorem 3.1 extends to Neumann problems with nonhomogeneous differential operators satisfying $\mathrm{H}(a)$, the property known for Dirichlet problems (see 8]) and for Neumann problems with $p$-Laplacian (see [18]). We also provide a new simpler proof which applies to all previous cases. Here the main idea is to apply the Lagrange multiplier rule in an $L^{r}(\Omega)$ related to the Sobolev embedding theorem and not in $W^{1, p}(\Omega)$. Thus we avoid working simultaneously with two terms in the form of divergence involving the solution.

The rest of the paper is organized as follows. Section 2 sets forth mathematical background and hypothesis $\mathrm{H}(a)$. Section 3 is devoted to the $W^{1, p}$ and $C^{1}$ minimizers. Section 4 presents our result on multiple solutions for problem (1.1).

\section{Mathematical BACKGRound AND the hypotheses on $a(x, y)$}

In the study of problem (1.1) we use two spaces, $C_{n}^{1}(\bar{\Omega})=\left\{u \in C^{1}(\bar{\Omega}): \frac{\partial u}{\partial n}=0\right\}$ and $W_{n}^{1, p}(\Omega)=\overline{C_{n}^{1}(\bar{\Omega})}\|\cdot\|$, where $\|\cdot\|$ is the usual Sobolev norm of $W^{1, p}(\Omega)$. We note that the cone $C_{+}=\left\{u \in C_{n}^{1}(\bar{\Omega}): u(x) \geq 0\right.$ for all $\left.x \in \bar{\Omega}\right\}$ has a nonempty interior given by int $C_{+}=\left\{u \in C_{+}: u(x)>0\right.$ for all $\left.x \in \bar{\Omega}\right\}$.

Consider the following nonlinear eigenvalue problem:

$$
\begin{cases}-\Delta_{p} u(x)=\lambda|u(x)|^{p-2} u(x) & \text { in } \Omega, \\ \frac{\partial u}{\partial n}=0 & \text { on } \partial \Omega .\end{cases}
$$

Recall that $\Delta_{p} u=\operatorname{div}\left(\|\nabla u\|^{p-2} \nabla u\right), p>1$ (the $p$-Laplacian). We know that $\lambda_{0}=0$ is the smallest eigenvalue of (2.1) with corresponding eigenspace $\mathbb{R}$. Let $\hat{u}_{0}$ be the $L^{p}$-normalized eigenfunction corresponding to 0 , so $\hat{u}_{0} \equiv 1 /\left(|\Omega|_{N}^{1 / p}\right)$ (here $|\cdot|_{N}$ is the Lebesgue measure on $\left.\mathbb{R}^{N}\right)$. The Ljusternik-Schnirelman theory, in addition to $\lambda_{0}$, produces a whole increasing sequence $\left\{\lambda_{n}\right\}_{n \geq 0}$ of eigenvalues such that $\lambda_{n} \rightarrow+\infty$. If $p=2$ (the linear eigenvalue problem), these are all the eigenvalues. If $p \neq 2$ (the nonlinear eigenvalue problem), we do not know if this is the case. Since $\lambda_{0}=0$ is isolated and the set $\sigma_{p}$ of all eigenvalues of (2.1) is closed, $\lambda_{1}=\inf \left[\lambda>0: \lambda \in \sigma_{p}\right]$ 
is the second eigenvalue of (2.1). We will use the following characterization of $\lambda_{1}$ (see [1]).

Proposition 2.1. There holds

$$
\lambda_{1}=\inf _{\hat{\gamma} \in \hat{\Gamma}} \max _{-1 \leq t \leq 1}\|\nabla \hat{\gamma}(t)\|_{p}^{p}
$$

where $\hat{\Gamma}=\left\{\hat{\gamma} \in C([-1,1], M): \hat{\gamma}(-1)=-\hat{u}_{0}, \hat{\gamma}(1)=\hat{u}_{0}\right\}, M=W_{n}^{1, p}(\Omega) \cap \partial B_{1}^{L^{p}}$ and $\partial B_{1}^{L^{p}}=\left\{u \in L^{p}(\Omega):\|u\|_{p}=1\right\}$.

Throughout the paper, the hypotheses on $a(x, y)$ in problem (1.1) are:

$\mathrm{H}(a) \quad a(x, y)=h(x,\|y\|) y$ for all $(x, y) \in \bar{\Omega} \times \mathbb{R}^{N}$, where $h(x, t)>0$ for all $(x, t) \in \bar{\Omega} \times(0,+\infty)$, and

(i) $a \in C_{\text {loc }}^{0, \alpha}\left(\bar{\Omega} \times \mathbb{R}^{N}, \mathbb{R}^{N}\right) \cap C^{1}\left(\bar{\Omega} \times\left(\mathbb{R}^{N} \backslash\{0\}\right), \mathbb{R}^{N}\right)$ with $0<\alpha \leq 1$;

(ii) for every $x \in \bar{\Omega}$ and $y \in \mathbb{R}^{N} \backslash\{0\},\left\|D_{y} a(x, y)\right\| \leq c_{1}\|y\|^{p-2}$ with $c_{1}>0$, $1<p<+\infty$

(iii) for every $x \in \bar{\Omega}$ and $y \in \mathbb{R}^{N} \backslash\{0\},\left(D_{y} a(x, y) \xi, \xi\right)_{\mathbb{R}^{N}} \geq c_{0}\|y\|^{p-2}\|\xi\|^{2}$ for all $\xi \in \mathbb{R}^{N}$, with $c_{0}>0$.

Such conditions are used widely in the literature (see, e.g., [5, 6], 9], [14, 22]).

Example 2.2. The following mappings satisfy hypotheses $\mathrm{H}(a)$. Here $\theta \in C^{1}(\bar{\Omega})$ and $\theta(x)>0$ for all $x \in \bar{\Omega}$ :

(e $\left.e_{1}\right) a(x, y)=\theta(x)\|y\|^{p-2} y$, for $p>1$ (corresponds to the weighted $p$-Laplacian);

(e $\left.e_{2}\right) a(x, y)=\theta(x)\left[\|y\|^{p-2} y+\ln \left(1+\|y\|^{p-2}\right) y\right], p \geq 2$;

$\left(e_{3}\right)$ for $1<\tau \leq p \leq q$ and $\tau \neq 2$,

$$
a(x, y)= \begin{cases}\theta(x)\left[\|y\|^{p-2} y+\|y\|^{q-2} y\right] & \text { if }\|y\| \leq 1 \\ \theta(x)\left[\|y\|^{p-2} y+\frac{q-2}{\tau-2}\|y\|^{\tau-2} y-\frac{q-\tau}{\tau-2} y\right] & \text { if }\|y\|>1\end{cases}
$$

$\left(e_{4}\right) a(x, y)=\theta(x)\left(\|y\|^{p-2} y+c \frac{\|y\|^{p-2} y}{1+\|y\|^{p}}\right)$ (corresponds to the weighted sum of the $p$-Laplacian and a generalized mean curvature operator), with $0<c<$ $4 p(p-1)$ if $1<p<2$ and $0<c<\frac{4 p}{(p-1)^{2}}$ if $p \geq 2$.

The function $G(x, y)$ determined by $\nabla_{y} G(x, y)=a(x, y)$ and $G(x, 0)=0$ for all $(x, y) \in \bar{\Omega} \times \mathbb{R}^{N}$ will be useful in our variational approach. Explicitly, $G(x, y)=$ $\int_{0}^{\|y\|} h(x, t) t d t$ for all $(x, y) \in \bar{\Omega} \times \mathbb{R}^{N}$. Hereafter, $\langle\cdot, \cdot\rangle$ denotes the duality brackets for the pair $\left(W_{n}^{1, p}(\Omega)^{*}, W_{n}^{1, p}(\Omega)\right)$ and $A: W_{n}^{1, p}(\Omega) \rightarrow W_{n}^{1, p}(\Omega)^{*}$ stands for the map

$$
\langle A(u), y\rangle=\int_{\Omega}(a(x, \nabla u), \nabla y)_{\mathbb{R}^{N}} d x \text { for all } u, y \in W_{n}^{1, p}(\Omega) .
$$

\section{3. $W_{n}^{1, p}$ VERSUS $C_{n}^{1}$ LOCAL MINIMIZERS}

In this section we prove a result which is an important tool for the proof of Theorem 4.2, It relates local $C_{n}^{1}(\bar{\Omega})$ - and $W_{n}^{1, p}(\Omega)$-minimizers of a subcritical $C^{1}$ functional. A result of this type was first proved for the Hilbert space $H_{0}^{1}(\Omega)$ in [3], and it was extended to $W_{0}^{1, p}(\Omega)$ in [8]. The $W_{n}^{1, p}(\Omega)$-version of the result can be found in [18. In the aforementioned works, $a(x, y)=a(y)=\|y\|^{p-2} y$. Here we extend the result to Neumann nonhomogeneous differential operators satisfying $\mathrm{H}(a)$. In addition, our proof is simpler, avoiding involved estimates conducted in [8] and [18. It relies on a new idea in the context of Lagrange multiplier rule, which applies for Dirichlet operators too. 
Let $f_{0}: \Omega \times \mathbb{R} \rightarrow \mathbb{R}$ be a Carathéodory function such that

$$
\left|f_{0}(x, s)\right| \leq \hat{a}(x)+\hat{c}|s|^{r-1} \text { for a.e. } x \in \Omega \text {, all } s \in \mathbb{R},
$$

with $\hat{a} \in L^{\infty}(\Omega)_{+}, \hat{c}>0,1 \leq r<p^{*}=\left\{\begin{array}{cl}\frac{N p}{N-p} & \text { if } N>p, \\ +\infty & \text { if } N \leq p .\end{array} \quad\right.$ Let $F_{0}(x, t)=$ $\int_{0}^{t} f_{0}(x, s) d s$ and define the $C^{1}$-functional $\varphi_{0}: W_{n}^{1, p}(\Omega) \rightarrow \mathbb{R}$ by

$$
\varphi_{0}(u)=\int_{\Omega} G(x, \nabla u(x)) d x-\int_{\Omega} F_{0}(x, u(x)) d x \quad \text { for all } u \in W_{n}^{1, p}(\Omega) .
$$

Theorem 3.1. If $u_{0} \in W_{n}^{1, p}(\Omega)$ is a local $C_{n}^{1}(\bar{\Omega})$-minimizer of $\varphi_{0}$, i.e., there exists $r_{0}>0$ such that

$$
\varphi_{0}\left(u_{0}\right) \leq \varphi_{0}\left(u_{0}+h\right) \text { for all } h \in C_{n}^{1}(\bar{\Omega}),\|h\|_{C_{n}^{1}(\bar{\Omega})} \leq r_{0},
$$

then $u_{0} \in C_{n}^{1}(\bar{\Omega})$ and it is a local $W_{n}^{1, p}(\Omega)$-minimizer of $\varphi_{0}$, i.e., there exists $r_{1}>0$ such that

$$
\varphi_{0}\left(u_{0}\right) \leq \varphi_{0}\left(u_{0}+h\right) \text { for all } h \in W_{n}^{1, p}(\Omega),\|h\| \leq r_{1} .
$$

Proof. Let an arbitrary $h \in C_{n}^{1}(\bar{\Omega})$ and let $t>0$ small. Then $\varphi_{0}\left(u_{0}\right) \leq \varphi_{0}\left(u_{0}+t h\right)$ and so $\left\langle\varphi_{0}^{\prime}\left(u_{0}\right), h\right\rangle \geq 0$. Because $C_{n}^{1}(\bar{\Omega})$ is dense in $W_{n}^{1, p}(\Omega)$, we get $\varphi_{0}^{\prime}\left(u_{0}\right)=0$. It follows that $A\left(u_{0}\right)=N_{0}\left(u_{0}\right)$, where $A$ is defined by (2.2) and $N_{0}(u)(\cdot)=f_{0}(\cdot, u(\cdot))$ for all $u \in W_{n}^{1, p}(\Omega)$, which can be expressed as

$$
\begin{cases}-\operatorname{div} a\left(x, \nabla u_{0}(x)\right)=f_{0}\left(x, u_{0}(x)\right) & \text { in } \Omega, \\ \frac{\partial u_{0}}{\partial n}=0 & \text { on } \partial \Omega\end{cases}
$$

(see [19]). By the Moser iteration process (see, e.g., [10, pp. 737-738]), we show that $u_{0} \in L^{\infty}(\Omega)$, and then by [11, Theorem 2] we have $u_{0} \in C^{1, \beta}(\bar{\Omega}) \cap C_{n}^{1}(\bar{\Omega})$, $0<\beta<1$.

Arguing by contradiction, suppose that $u_{0}$ is not a local $W_{n}^{1, p}(\Omega)$-minimizer of $\varphi_{0}$. Then, due to the Sobolev embedding theorem, we deduce for every $\varepsilon>0$ that

$$
\inf \left[\varphi_{0}\left(u_{0}+h\right): h \in \bar{B}_{\varepsilon}^{r}\right]=: m_{\varepsilon}<\varphi_{0}\left(u_{0}\right),
$$

where $\bar{B}_{\varepsilon}^{r}=\left\{u \in W_{n}^{1, p}(\Omega):\|u\|_{r} \leq \varepsilon\right\}$ and $\|\cdot\|_{r}$ denotes the $L^{r}(\Omega)$-norm. Here, we have chosen $r \in\left[p, p^{*}\right)$ satisfying (3.1). We claim that we can find $h_{\varepsilon} \in \bar{B}_{\varepsilon}^{r}$ such that $m_{\varepsilon}=\varphi_{0}\left(u_{0}+h_{\varepsilon}\right)$. To this end, let $\left\{h_{n}\right\}_{n \geq 1}$ be a minimizing sequence for (3.2). By $\mathrm{H}(a)$ we get

$$
\frac{c_{0}}{p(p-1)}\|y\|^{p} \leq G(x, y) \leq \frac{c_{1}}{p(p-1)}\|y\|^{p} \text { for all }(x, y) \in \bar{\Omega} \times \mathbb{R}^{N} .
$$

By (3.1), (3.3) and since $\left\{h_{n}\right\}_{n \geq 1} \subset \bar{B}_{\varepsilon}^{r}$, we see that $\left\{h_{n}\right\}_{n \geq 1}$ is bounded in $W_{n}^{1, p}(\Omega)$. We may assume that $h_{n} \stackrel{\mathrm{w}}{\rightarrow} h_{\varepsilon}$ in $W_{n}^{1, p}(\Omega)$ and $h_{n} \rightarrow h_{\varepsilon}$ in $L^{r}(\Omega)$ as $n \rightarrow \infty$. Then $h_{\varepsilon} \in \bar{B}_{\varepsilon}^{r}$ and $\varphi_{0}\left(u_{0}+h_{\varepsilon}\right) \leq \liminf _{n \rightarrow \infty} \varphi_{0}\left(u_{0}+h_{n}\right)=m_{\varepsilon}$, because $\varphi_{0}$ is sequentially weakly lower semicontinuous. Therefore the claim is true, and (3.2) yields

$$
\varphi_{0}\left(u_{0}+h_{\varepsilon}\right)<\varphi_{0}\left(u_{0}\right) .
$$

By virtue of the Lagrange multiplier rule, we can find $\lambda_{\varepsilon} \leq 0$ such that

$$
\varphi_{0}^{\prime}\left(u_{0}+h_{\varepsilon}\right)=A\left(u_{0}+h_{\varepsilon}\right)-N_{0}\left(u_{0}+h_{\varepsilon}\right)=\lambda_{\varepsilon}\left|h_{\varepsilon}\right|^{r-2} h_{\varepsilon} .
$$

We set $v_{\varepsilon}=u_{0}+h_{\varepsilon}$. 
Case 1. $\lambda_{\varepsilon} \in[-1,0]$ for all $\varepsilon \in(0, \delta)$, with $\delta>0$.

Then (3.5) reads as

$$
-\operatorname{div} a\left(x, \nabla v_{\varepsilon}(x)\right)=f_{0}\left(x, v_{\varepsilon}(x)\right)+\lambda_{\varepsilon}\left|\left(v_{\varepsilon}-u_{0}\right)(x)\right|^{r-2}\left(v_{\varepsilon}-u_{0}\right)(x) .
$$

We derive that $v_{\varepsilon} \in L^{\infty}(\Omega)$ and $\left\|v_{\varepsilon}\right\|_{\infty} \leq M_{0}$ for all $\varepsilon \in(0, \delta)$, for some $M_{0}>0$. This follows by applying the Moser iteration technique. In view of $\mathrm{H}(a)$, by [11, Theorem 2], there exist $\theta \in(0,1)$ and $M_{1}>0$ such that

$$
v_{\varepsilon} \in C_{n}^{1, \theta}(\bar{\Omega}) \text { and }\left\|v_{\varepsilon}\right\|_{C_{n}^{1, \theta}(\bar{\Omega})} \leq M_{1} \text { for all } \varepsilon \in(0, \delta) .
$$

Case 2. $\lambda_{\varepsilon}<-1$ along a sequence $\varepsilon \downarrow 0$.

We set $a_{\varepsilon}(x, y)=\frac{1}{\left|\lambda_{\varepsilon}\right|} a(x, y)$. From (3.5) we have

$$
-\operatorname{div} a_{\varepsilon}\left(x, \nabla v_{\varepsilon}(x)\right)=\frac{1}{\left|\lambda_{\varepsilon}\right|} f_{0}\left(x, v_{\varepsilon}(x)\right)-\left|\left(v_{\varepsilon}-u_{0}\right)(x)\right|^{p-2}\left(v_{\varepsilon}-u_{0}\right)(x) .
$$

Again, $v_{\varepsilon} \in L^{\infty}(\Omega)$ and $\left\|v_{\varepsilon}\right\|_{\infty} \leq M_{0}$ for all $\varepsilon$, for some $M_{0}>0$. Via [11, Theorem 2], we find $\theta \in(0,1)$ and $M_{1}>0$ such that

$$
v_{\varepsilon} \in C_{n}^{1, \theta}(\bar{\Omega}) \text { and }\left\|v_{\varepsilon}\right\|_{C_{n}^{1, \theta}(\bar{\Omega})} \leq M_{1} \text { along a sequence } \varepsilon \downarrow 0 \text {. }
$$

Since $C_{n}^{1, \theta}(\bar{\Omega})$ is embedded compactly in $C_{n}^{1}(\bar{\Omega})$, by (3.6) and (3.7) we see that $u_{0}+h_{\varepsilon} \rightarrow \hat{y}$ in $C_{n}^{1}(\bar{\Omega})$ as $\varepsilon \rightarrow 0$. Since $h_{\varepsilon} \rightarrow 0$ in $L^{r}(\Omega)$, we get $\hat{y}=u_{0}$. Then our hypothesis gives $\varphi_{0}\left(u_{0}\right) \leq \varphi_{0}\left(u_{0}+h_{\varepsilon}\right)$ for $\varepsilon>0$ small, which contradicts (3.4).

\section{Three SOLUtions THEOREM}

In this section we establish the existence of three nontrivial smooth solutions for problem (1.1). The hypotheses on $f(x, s)$ are the following:

$\mathrm{H}(f) f: \Omega \times \mathbb{R} \rightarrow \mathbb{R}$ is a Carathéodory function such that $f(x, 0)=0$ a.e. in $\Omega$ :

(i) $|f(x, s)| \leq a(x)+c|s|^{r-1}$ for a.a. $x \in \Omega$, all $s \in \mathbb{R}$, with $a \in L^{\infty}(\Omega)_{+}, c>0$, $1 \leq r<p^{*}$

(ii) if $F(x, t)=\int_{0}^{t} f(x, s) d s$, there exists $\theta \in L^{\infty}(\Omega), \theta \leq 0, \theta \neq 0$, such that

$$
\limsup _{|s| \rightarrow \infty} \frac{p F(x, s)}{|s|^{p}} \leq \theta(x) \text { uniformly for a.a. } x \in \Omega ;
$$

(iii) there exist $\delta_{0}>0$ and $\eta>\lambda_{1}$ such that

$$
\frac{c_{1} \eta}{p(p-1)}|s|^{p} \leq F(x, s) \text { for a.a. } x \in \Omega \text {, all }|s| \leq \delta_{0}
$$

(iv) there exists $\lambda>0$ such that

$$
\left(f(x, s)+\lambda|s|^{p-2} s\right) s \geq 0 \text { for a.a. } x \in \Omega, \text { all } s \in \mathbb{R} .
$$

Example 4.1. The following function satisfies hypotheses $\mathrm{H}(f)$ (for the sake of simplicity, we drop the $x$-dependence):

$$
f(s)= \begin{cases}\hat{\eta}|s|^{p-2} s & \text { if }|s| \leq 1, \\ c|s|^{q-2} s-(c-\hat{\eta})|s|^{p-2} s & \text { if }|s|>1,\end{cases}
$$

with $\hat{\eta}>\frac{c_{1} \lambda_{1}}{p-1}, c>\hat{\eta}$ and $1<q<p$.

Theorem 4.2. If hypotheses $\mathrm{H}(a)$ and $\mathrm{H}(f)$ hold, then problem (1.1) has at least three nontrivial smooth solutions: $u_{0} \in \operatorname{int} C_{+}, v_{0} \in-\operatorname{int} C_{+}$and $y_{0} \in C_{n}^{1}(\bar{\Omega})$. 
Proof. First using truncations and the direct method, we produce two nontrivial smooth solutions of opposite constant sign. For $\lambda>0$ as in $\mathrm{H}(f)$ (iv), let

$$
f_{+}^{\lambda}(x, s)= \begin{cases}0 & \text { if } s \leq 0 \\ f(x, s)+\lambda s^{p-1} & \text { if } s>0\end{cases}
$$

Let $F_{+}^{\lambda}(x, t)=\int_{0}^{t} f_{+}^{\lambda}(x, s) d s$ and define the $C^{1}$-functional $\varphi_{+}^{\lambda}: W_{n}^{1, p}(\Omega) \rightarrow \mathbb{R}$ by

$$
\varphi_{+}^{\lambda}(u)=\int_{\Omega} G(x, \nabla u) d x+\frac{\lambda}{p}\|u\|_{p}^{p}-\int_{\Omega} F_{+}^{\lambda}(x, u) d x \text { for all } u \in W_{n}^{1, p}(\Omega) .
$$

By hypotheses $\mathrm{H}(f)$ (i) and (ii), given $\varepsilon>0$, we find $a_{\varepsilon} \in L^{\infty}(\Omega)_{+}$such that

$$
F(x, s) \leq \frac{1}{p}(\theta(x)+\varepsilon)|s|^{p}+a_{\varepsilon}(x) \text { for a.a. } x \in \Omega \text {, all } s \in \mathbb{R} .
$$

Then (3.3), (4.1) and Lemma 2 of 1 imply for all $u \in W_{n}^{1, p}(\Omega)$ that

$$
\begin{aligned}
\varphi_{+}^{\lambda}(u) & \geq \int_{\Omega} G(x, \nabla u) d x-\frac{1}{p} \int_{\Omega} \theta|u|^{p} d x-\frac{\varepsilon}{p}\|u\|^{p}-c_{2} \\
& \geq \frac{c_{0}}{p(p-1)}\|\nabla u\|_{p}^{p}-\frac{1}{p} \int_{\Omega} \theta|u|^{p} d x-\frac{\varepsilon}{p}\|u\|^{p}-c_{2} \geq \frac{\xi_{0}-\varepsilon}{p}\|u\|^{p}-c_{2},
\end{aligned}
$$

with constants $c_{2}, \xi_{0}>0$. For $\varepsilon \in\left(0, \xi_{0}\right)$, we infer that $\varphi_{+}^{\lambda}$ is coercive. Since $\varphi_{+}^{\lambda}$ is sequentially weakly lower semicontinuous, there exists $u_{0} \in W_{n}^{1, p}(\Omega)$ such that

$$
\varphi_{+}^{\lambda}\left(u_{0}\right)=\inf \varphi_{+}^{\lambda}=: m_{+}^{\lambda} .
$$

If $\xi \in\left(0, \delta_{0}\right]$, by hypothesis $\mathrm{H}(f)$ (iii) we have that $\varphi_{+}^{\lambda}(\xi)<0$, and so $\varphi_{+}^{\lambda}\left(u_{0}\right)<0$, which ensures that $u_{0} \neq 0$. Also there holds $\left(\varphi_{+}^{\lambda}\right)^{\prime}\left(u_{0}\right)=0$, that is,

$$
A\left(u_{0}\right)+\lambda\left|u_{0}\right|^{p-2} u_{0}=f_{+}^{\lambda}\left(x, u_{0}\right) .
$$

Acting on (4.3) with $-u_{0}^{-} \in W_{n}^{1, p}(\Omega)$, we obtain $u_{0} \geq 0$. Consequently, $u_{0}$ solves problem (1.1), therefore $u_{0} \in C_{+} \backslash\{0\}$ (see [11]), while hypothesis $\mathrm{H}(f)$ (iv) implies

$$
-\operatorname{div} a\left(x, \nabla u_{0}(x)\right)+\lambda u_{0}(x)^{p-1}=f\left(x, u_{0}(x)\right)+\lambda u_{0}(x)^{p-1} \geq 0 \text { a.e. in } \Omega .
$$

From the maximum principle of [14] (see also [6, 22]) and (4.4), we get $u_{0} \in \operatorname{int} C_{+}$.

In a similar fashion, using the truncation

$$
f_{-}^{\lambda}(x, s)= \begin{cases}f(x, s)+\lambda|s|^{p-2} s & \text { if } s<0, \\ 0 & \text { if } s \geq 0,\end{cases}
$$

let $F_{-}^{\lambda}(x, t)=\int_{0}^{t} f_{-}^{\lambda}(x, s) d s$ and define the $C^{1}$-functional $\varphi_{-}^{\lambda}: W_{n}^{1, p}(\Omega) \rightarrow \mathbb{R}$ by

$$
\varphi_{-}^{\lambda}(u)=\int_{\Omega} G(x, \nabla u) d x+\frac{\lambda}{p}\|u\|_{p}^{p}-\int_{\Omega} F_{-}^{\lambda}(x, u) d x \text { for all } u \in W_{n}^{1, p}(\Omega) .
$$

Then via the direct method we obtain a nontrivial smooth solution $v_{0} \in-\operatorname{int} C_{+}$.

Let $\varphi: W_{n}^{1, p}(\Omega) \rightarrow \mathbb{R}$ be the $C^{1}$-energy functional for problem (1.1) defined by

$$
\varphi(u)=\int_{\Omega} G(x, \nabla u) d x-\int_{\Omega} F(x, u) d x \text { for all } u \in W_{n}^{1, p}(\Omega) .
$$

Note that $\left.\varphi\right|_{C_{+}}=\left.\varphi_{+}^{\lambda}\right|_{C_{+}}$, and so $u_{0} \in \operatorname{int} C_{+}$is a local $C_{n}^{1}(\bar{\Omega})$-minimizer of $\varphi$. On the basis of Theorem 3.1, we infer that $u_{0}$ is a local $W_{n}^{1, p}(\Omega)$-minimizer of $\varphi$. Similarly, we have that $v_{0} \in-\operatorname{int} C_{+}$is a local $W_{n}^{1, p}(\Omega)$-minimizer of $\varphi$. We may 
assume that $\varphi$ has a finite critical set and $\varphi\left(v_{0}\right) \leq \varphi\left(u_{0}\right)$. Reasoning as in [15] (the proof of Proposition 6), we can find $\rho>0$ small such that

$$
\varphi\left(v_{0}\right) \leq \varphi\left(u_{0}\right)<\inf \left[\varphi(u):\left\|u-u_{0}\right\|=\rho\right]=: m_{\rho} .
$$

Moreover, from (4.1) and 1, Lemma 2], we have that $\varphi$ is coercive; hence it satisfies the Palais-Smale condition. Then, through (4.5), the mountain pass theorem (see, e.g., [10, p. 648]) provides $y_{0} \in W_{n}^{1, p}(\Omega)$ with $\varphi\left(y_{0}\right) \geq m_{\rho}$ and $\varphi^{\prime}\left(y_{0}\right)=0$. It follows that $y_{0} \in C_{0}^{1}(\bar{\Omega})$ is a solution of (1.1) and $y_{0} \notin\left\{u_{0}, v_{0}\right\}$ (see (4.5)).

We need to show that $y_{0} \neq 0$. By the mountain pass theorem we have

$$
\varphi\left(y_{0}\right)=\inf _{\gamma \in \Gamma} \max _{t \in[0,1]} \varphi(\gamma(t)),
$$

where $\Gamma=\left\{\gamma \in C\left([0,1], W_{n}^{1, p}(\Omega)\right): \gamma(0)=v_{0}, \gamma(1)=u_{0}\right\}$. If we can produce a path $\gamma_{0} \in \Gamma$ such that $\left.\varphi\right|_{\gamma_{0}}<0$, then from (4.6) we have $\varphi\left(y_{0}\right)<0=\varphi(0)$, and so $y_{0} \neq 0$. In what follows we generate such a path $\gamma_{0} \in \Gamma$. Let $M=W_{n}^{1, p}(\Omega) \cap \partial B_{1}^{L^{p}}$ as in Proposition 2.1. endowed with the relative $W_{n}^{1, p}(\Omega)$-topology. Let $M_{c}=$ $M \cap C_{n}^{1}(\bar{\Omega})$ be furnished with the relative $C_{n}^{1}(\bar{\Omega})$-topology. Evidently, $M_{c}$ is dense in $M$. So, $\hat{\Gamma}_{c}=\left\{\hat{\gamma} \in C\left([-1,1], M_{c}\right): \hat{\gamma}(-1)=-\hat{u}_{0}, \hat{\gamma}(1)=\hat{u}_{0}\right\}$ is dense in $\hat{\Gamma}$ introduced in Proposition 2.1] Then, from Proposition 2.1, we see that given $\varepsilon>0$ we can find $\hat{\gamma}_{0} \in \hat{\Gamma}_{c}$ with the property

$$
\max \left[\|\nabla u\|_{p}^{p}: u \in \hat{\gamma}_{0}([-1,1])\right] \leq \lambda_{1}+\varepsilon .
$$

If $\eta>\lambda_{1}$ is as in $\mathrm{H}(f)$ (iii), let us choose $\varepsilon>0$ such that $\lambda_{1}+\varepsilon<\eta$. Also let $\xi>0$ be small such that $\xi|u(x)| \leq \delta_{0}$ for all $x \in \bar{\Omega}$ and all $u \in \hat{\gamma}_{0}([-1,1])$, with $\delta_{0}>0$ in $\mathrm{H}(f)$ (iii). Then, by (3.3), $\mathrm{H}(f)$ (iii), and (4.7), we have

$$
\varphi(\xi u) \leq \frac{c_{1} \xi^{p}}{p(p-1)}\|\nabla u\|_{p}^{p}-\frac{c_{1} \eta \xi^{p}}{p(p-1)} \leq \frac{c_{1} \xi^{p}}{p(p-1)}\left(\lambda_{1}+\varepsilon-\eta\right)<0
$$

for all $u \in \hat{\gamma}_{0}([-1,1])$. Therefore, $\bar{\gamma}_{0}=\xi \hat{\gamma}_{0}$ is a path from $-\xi \hat{u}_{0}$ to $\xi \hat{u}_{0}$ that satisfies

$$
\left.\varphi\right|_{\bar{\gamma}_{0}}<0 \text {. }
$$

Recall that $m_{+}^{\lambda}=\varphi_{+}^{\lambda}\left(u_{0}\right)<0=\varphi_{+}^{\lambda}(0)$. We may assume that $\left\{0, u_{0}\right\}$ are the only critical points of $\varphi_{+}^{\lambda}$. Indeed, if $y \in W_{n}^{1, p}(\Omega)$ is another critical point of $\varphi_{+}^{\lambda}$, then as for $u_{0}$ we show that $y \in \operatorname{int} C_{+}$. Thus it is a third nontrivial smooth solution of (1.1) and we are done. Hence, we can apply to $\varphi_{+}^{\lambda}$ the Second Deformation Theorem (see, e.g., [10, p. 628]) and obtain a homotopy $h:[0,1] \times\left(\left\{u \in W_{n}^{1, p}(\Omega)\right.\right.$ : $\left.\left.\varphi_{+}^{\lambda}(u) \leq 0\right\} \backslash\{0\}\right) \rightarrow\left\{u \in W_{n}^{1, p}(\Omega): \varphi_{+}^{\lambda}(u) \leq 0\right\}$ satisfying

(a) $h(0, \cdot)$ is the identity map;

(b) $h(1, \cdot)$ has the range $\left\{u_{0}\right\}$ (due to (4.2));

(c) $\varphi_{+}^{\lambda}(h(\cdot, u))$ is nonincreasing on $[0,1]$ for all $u$.

Let $\gamma_{+}(t)=h\left(t, \xi \hat{u}_{0}\right)$ for all $t \in[0,1]$. Then (a) shows that $\gamma_{+}(0)=\xi \hat{u}_{0}$, while (b) implies $\gamma_{+}(1)=u_{0}$. From (c) and (4.8) we get

$$
\varphi_{+}^{\lambda}\left(\gamma_{+}(t)\right)=\varphi_{+}^{\lambda}\left(h\left(t, \xi \hat{u}_{0}\right)\right) \leq \varphi_{+}^{\lambda}\left(\xi \hat{u}_{0}\right)<0 \text { for all } t \in[0,1] .
$$

By $\mathrm{H}(f)($ iv $), s=0$ is a global minimizer of $s \mapsto F(x, s)+\frac{\lambda}{p}|s|^{p}$ for a.a. $x \in \Omega$, so

$$
\begin{aligned}
\varphi(u)= & \int_{\Omega} G(x, \nabla u) d x+\frac{\lambda}{p}\|u\|_{p}^{p}-\int_{\Omega}\left[F\left(x, u^{+}\right)+\frac{\lambda}{p}\left(u^{+}\right)^{p}\right] d x \\
& -\int_{\Omega}\left[F\left(x,-u^{-}\right)+\frac{\lambda}{p}\left(u^{-}\right)^{p}\right] d x \leq \varphi_{+}^{\lambda}(u) \text { for all } u \in W_{n}^{1, p}(\Omega) .
\end{aligned}
$$


Combining with (4.9), we see that $\gamma_{+}$is a path joining $\xi \hat{u}_{0}$ and $u_{0}$ that satisfies $\left.\varphi\right|_{\gamma_{+}}<0$. In a similar fashion we produce a path $\gamma_{-}$joining $v_{0}$ and $-\xi \hat{u}_{0}$ such that $\left.\varphi\right|_{\gamma_{-}}<0$. Recalling (4.8), we concatenate $\gamma_{-}, \bar{\gamma}_{0}$ and $\gamma_{+}$to construct a path $\gamma_{0} \in \Gamma$ satisfying $\left.\varphi\right|_{\gamma_{0}}<0$. We conclude that $y_{0} \in C_{n}^{1}(\bar{\Omega})$ is a third nontrivial solution of (1.1), which completes the proof.

\section{ACKNOWLEDGEMENT}

The authors are grateful to the editor and the three referees for their comments.

\section{REFERENCES}

1. S. Aizicovici, N. S. Papageorgiou and V. Staicu, The spectrum and an index formula for the Neumann p-Laplacian and multiple solutions for problems with crossing nonlinearities, Discrete Cont. Dyn. Syst. 25 (2009), 431-456. MR2525184 (2010h:35430)

2. G. Bonanno and P. Candito, Three solutions to a Neumann problem for elliptic equations involving the p-Laplacian. Arch. Math. (Basel) 80 (2003), 424-429. MR1982841(2004h:35061)

3. H. Brezis and L. Nirenberg, $H^{1}$ versus $C^{1}$ local minimizers. C. R. Acad. Sci Paris 317 (1993), 465-472. MR1239032 (94g:49044)

4. S. Carl and K. Perera, Sign-changing and multiple solutions for the p-Laplacian, Abstr. Appl. Anal. 7 (2002), 613-625. MR 1950611 (2004a:35061)

5. Ph. Clément, M. Garcia-Huidobro, R. Manasevich, and K. Schmitt, Mountain pass type solutions for quasilinear elliptic equations, Calc. Var. Partial Diff. Equ. 11 (2000), 33-62. MR:1777463(2001h:35051)

6. L. Damascelli, Comparison theorems for some quasilinear degenerate elliptic operators and applications to symmetry and monotonicity results, Ann. Inst. H. Poincaré Anal. Non Linéaire 15 (1998), 493-516. MR1632933 (99e:35081)

7. F. Faraci, Multiple solutions for two nonlinear problems involving the p-Laplacian, Nonlinear Anal. 63 (2005), e1017-e1029 (electronic).

8. J. Garcia Azorero, I. Peral Alonso, and J. Manfredi, Sobolev versus Hölder local minimizers and global multiplicity for some quasilinear elliptic equations, Commun. Contemp. Math. 2 (2000), 385-404. MR:1776988 (2001k:35062)

9. M. Garcia-Huidobro, R. Manasevich, and P. Ubilla, Existence of positive solutions for some Dirichlet problems with an asymptotically linear operator, Electron. J. Differential Equations 1995, No. 10 (electronic). MR1345249 (96f:35053)

10. L. Gasiński and N. S. Papageorgiou, Nonlinear Analysis, Chapman \& Hall/CRC, Boca Raton, FL, 2006. MR.2168068 (2006e:47001)

11. G. M. Lieberman, Boundary regularity for solutions of degenerate elliptic equations, Nonlinear Anal. 12 (1988), 1203-1219. MR969499 (90a:35098)

12. S. Liu, Multiple solutions for coercive p-Laplacian equations, J. Math. Anal. Appl. 316 (2006), 229-236. MR2201759 (2006m:35103)

13. J. Liu and S. Liu, The existence of multiple solutions to quasilinear elliptic equations, Bull. London Math. Soc. 37 (2005), 592-600. MR2143739 (2006m:35102)

14. M. Montenegro, Strong maximum principles for supersolutions of quasilinear elliptic equations, Nonlinear Anal. 37 (1999), 431-448. MR1691019 (2000i:35048)

15. D. Motreanu, V. V. Motreanu and N. S. Papageorgiou, A degree theoretic approach for multiple solutions of constant sign for nonlinear elliptic equations, Manuscripta Math. 124 (2007), 507-531. MR.2357796 (2009f:35100)

16. D. Motreanu, V. V. Motreanu and N. S. Papageorgiou, Multiple nontrivial solutions for nonlinear eigenvalue problems, Proc. Amer. Math. Soc. 135 (2007), 3649-3658. MR2336581 (2008m:35259)

17. D. Motreanu, V. V. Motreanu and N. S. Papageorgiou, A unified approach for multiple constant sign and nodal solutions, Adv. Differential Equations 12 (2007), 1363-1392. MR 2382729 (2009b:35142)

18. D. Motreanu, V. V. Motreanu and N. S. Papageorgiou, Nonlinear Neumann problems near resonance, Indiana Univ. Math. J. 58 (2009), 1257-1279. MR2541367|(2010e:35101)

19. D. Motreanu and N. S. Papageorgiou, Existence and multiplicity of solutions for Neumann problems, J. Differential Equations 232 (2007), 1-35. MR2281188(2007h:35046) 
20. E. Papageorgiou and N. S. Papageorgiou, A multiplicity theorem for problems with the p-Laplacian, J. Functional Anal. 244 (2007), 63-77. MR2294475 (2008e:35074)

21. X. Wu and K. K. Tan, On existence and multiplicity of solutions of Neumann boundary value problems for quasi-linear elliptic equations, Nonlinear Anal. 65 (2006), 1334-1347. MR2245508 (2007d:35111)

22. Q. Zhang, A strong maximum principle for differential equations with nonstandard $p(x)$ growth conditions, J. Math. Anal. Appl. 312 (2005), 24-32. MR2175201 (2006e:35132)

23. Z. Zhang, J. Chen and S. Li, Construction of a pseudo-gradient vector field and sign-changing multiple solutions involving the p-Laplacian, J. Differential Equations 201 (2004), 287-303. MR:2059609 (2006d:35047)

Département de Mathématiques, Université de Perpignan, 66860 Perpignan, France

E-mail address: motreanu@univ-perp.fr

Department of Mathematics, National Technical University, Athens 15780, Greece

E-mail address: npapg@math.ntua.gr 